\title{
SUBSTANTIAL REDUCTION OF CORTICAL NORADRENALINE BY LESIONS OF ADRENERGIC PATHWAY DOES NOT PREVENT EFFECTS OF MONOCULAR DEPRIVATION ${ }^{1}$
}

\author{
N. W. DAW, ${ }^{2}$ T. W. ROBERTSON,* R. K. RADER, T. O. VIDEEN, AND C. J. COSCIA \\ Department of Physiology and Biophysics and McDonnell Center for Study of Higher Brain Functions, Washington University \\ School of Medicine, St. Louis, Missouri 63110, *School of Optometry, University of Missouri, St. Louis, Missouri 63121, and \\ $\ddagger$ E. A. Doisy Department of Biochemistry, St. Louis University School of Medicine, St. Louis, Missouri 63104
}

Received October 19, 1983; Accepted December 13, 1983

\begin{abstract}
We tested the theory that depletion of noradrenaline reduces the plasticity of the visual cortex in kittens by using another method of depletion. Lesions were made in the lateral hypothalamus to interrupt fibers in the dorsal noradrenergic bundle going from the locus ceruleus to the telencephalon. The lesions were induced at approximately $3 \frac{1}{2}$ weeks of age in kittens; approximately 2 weeks later one eye was sutured shut, and about 10 days after that cells were recorded in the visual cortex. The location of the lesions was verified histologically, and the effect of the lesions was verified by noradrenaline analyses (high pressure liquid chromatography-electrochemistry) of samples from the visual cortex.

The noradrenaline content of the visual cortex was reduced by 70 to $90 \%$. However, the majority of cells recorded in the visual cortex could not be driven through the eye that had been sutured closed. The ocular dominance histograms for cells in the visual cortex were indistinguishable from those of kittens that were monocularly deprived for a similar period in the "critical period" and that had normal amounts of noradrenaline in their visual cortex. Therefore, we conclude that reduction of the noradrenaline content of the visual cortex by 70 to $90 \%$ is insufficient, by itself, to prevent the physiological changes that occur in the visual cortex after monocular deprivation.
\end{abstract}

Properties of cells in the visual cortex of the kitten can be altered permanently by restricting the visual input. The classic example of this is monocular deprivation, where the eyelids of one eye are sutured shut for some time during a "critical period," which lasts from eye opening to several months of age in the kitten (Hubel and Wiesel, 1970). One result of this procedure is that cells in the visual cortex become dominated by the open eye, and very few of them can be driven through the sutured eye when it is reopened for the experiment (Wiesel and Hubel, 1963; for recent summaries see Movshon and van Sluyters, 1981; Sherman and Spear, 1982). Kasamatsu and Pettigrew $(1976,1979)$ have hypothe-

\footnotetext{
${ }^{1}$ This work was supported by National Institutes of Health Research Grant EY00053 and Program Project Grant NS15070. T. V. was supported by the McDonnell Center for Studies of Higher Brain Function and National Institutes of Health Training Grant EY07057. We thank Elizabeth Coscia for carrying out the high pressure liquid chromatography measurements, Jeanette Cohen for the histology, and Janice Wuelling for typing the manuscript. Dr. G. F. Wooten kindly analyzed the depletion of noradrenaline in some pilot experiments.

${ }^{2}$ To whom correspondence should be addressed.
}

sized that noradrenaline (NA) plays an important role in this plasticity of the kitten visual cortex. They have found that depletion of noradrenaline by administration of 6-hydroxydopamine (6-OHDA) into the lateral ventricle or directly into the cortex (Kasamatsu et al., 1979) prevents this effect; that is, after 6-OHDA treatment as well as eye suture, cells in the visual cortex can still be driven through the sutured eye. Infusion of NA directly into the cortex reverses the effect of 6-OHDA (Pettigrew and Kasamatsu, 1978; Kasamatsu et al., 1979).

The interpretation of these experiments was questioned by Bear and Daniels (1983), who found that neonatal intraperitoneal administration of 6-OHDA also reduces the NA content of the visual cortex but does not alter the physiological effects of suturing one eye. Therefore, we decided to use another method to deplete NA: lesions of the dorsal noradrenergic bundle (DNAB). All noradrenergic fibers going to the cerebral cortex arise in the locus ceruleus. They project rostrally through the brainstem and lateral hypothalamus, forming the DNAB, then dorsally through the frontal cortex and posteriorly to the visual cortex (Maeda et al., 1973; Chu and Bloom, 1974; Morrison et al., 1981). We chose the lateral hypo- 
thalamus as a site for the lesions because of its distance from the visual cortex and brainstem areas, to avoid direct disruption of visual or vital functions. At this point the fibers have coalesced and do not cross the midline on their way to the telencephalon. All lesions were unilateral, to avoid feeding and drinking problems (Anand and Brobeck, 1951; Teitelbaum and Epstein, 1962; Wolgin and Teitelbaum, 1978). Some lesions were electrolytic, to avoid the use of 6-OHDA and to ensure that they were unilateral. Hence, the NA content on one side of the visual cortex could be compared with the opposite side in the same animal. Other unilateral lesions were made by infusion of small quantities of 6-OHDA directly into the DNAB, to produce a lesion with a more selective effect on the NA fibers passing through the area.

\section{Materials and Methods}

Lesions and eye suture. The electrolytic lesions were made with a sharpened stainless steel needle, insulated to about $1.2 \mathrm{~mm}$ from the tip. A DC current of $200 \mu \mathrm{A}$ was passed at each position for $60 \mathrm{sec}$, frequently repeated after an interval. This produced a hole of about 1 $\mathrm{mm}$ in diameter and $1.5 \mathrm{~mm}$ in length in the tissue. Pilot experiments showed that a large lesion was necessary to produce substantial depletion of NA in the cortex. Therefore, a series of lesions was made at A6 to A7 between $\mathrm{L} 1$ and L5 and between U1.5 and D3.5, with the vertical extent of the lesion smaller at more lateral positions.

6-OHDA lesions were made with a syringe (Hamilton, Reno, NV) inserted at A7, L3, D1. Two microliters of a $3 \mu \mathrm{g} / \mu \mathrm{l}$ solution were injected over $10 \mathrm{~min}$ for a total dose of $6 \mu \mathrm{g}$.

Lesions and eye suture were done under halothane anesthesia. Eye suture was done in the usual way (Wiesel and Hubel, 1963; Berman and Daw, 1977). The lesions were always made on the left side, and the cortex was always recorded on the left side, except in cases where the opposite side was recorded as a control. Since there is a bias in favor of the contralateral eye in ocular dominance in the cortex, half of the animals had the left eye sutured and half had the right eye sutured.

Recording procedures. These were conventional (Wiesel and Hubel, 1963; Rerman and Daw, 1977). The animal was anesthetized with halothane, with a tracheotomy for respiration, an intravenous cannula for delivery of fluids and paralytic (Pavulon, Organon Inc., NJ), and contact lenses to prevent the cornea from drying. After a cell was isolated, it was characterized according to ocular dominance (Groups 1 to 7 of Hubel and Wiesel, 1959), orientation preference, direction preference, velocity preference, length specificity, type (simple or complex), spontaneous activity, and other characteristics as appropriate. Particular attention was paid to ocular dominance; at least two experimenters were required to make a judgement on each cell. To avoid sampling bias from staying in the same ocular dominance column, the electrode was angled at $15^{\circ}$ to the vertical down the medial bank of the lateral gyrus, and it was moved $300 \mu \mathrm{m}$ after each cell for the first $1 \mathrm{~mm}$ and $150 \mu \mathrm{m}$ after each cell thereafter (Daw and Wyatt, 1976; Berman and Daw,
1977). At least three penetrations, spaced about $1 \mathrm{~mm}$ apart, were made in each animal. About 40 cells were recorded in each experiment.

$N A$ analyses. At the end of the recordings the bone and dura over the posterior cortex were opened up to take samples for biochemical assay. Three samples were taken on each side: one in the lateral gyrus near the level of recording (APO), one posterior to this in the postlateral gyrus, and one lateral to this in the suprasylvian gyrus. The samples were blotted with Kimwipes to remove excess blood and were immediately frozen on a brass block in dry ice. They were then stored at $-90^{\circ} \mathrm{F}$ until assayed.

Catecholamine analyses were performed by paired-ion reverse-phase high pressure liquid chromatography (Mitchell and Coscia, 1978) with electrochemical detection (Kissinger et al., 1973). Brain samples (10 to 150 $\mathrm{mg}$ ) were homogenized in $0.1 \mathrm{ml}$ of $0.4 \mathrm{M}$ perchloric acid in a 2 -ml glass Wheaton homogenizer. The homogenate was centrifuged in a Beckman microfuge for $5 \mathrm{~min}$, and the supernatant was analyzed directly. Routinely, samples were assayed immediately, although they were stable in this solution at $-70^{\circ} \mathrm{C}$ for several weeks.

The high pressure liquid chromatograph consisted of a Waters Associates solvent delivery system $(6000 \mathrm{~A})$ and universal loop injector (U6K) coupled to a Bioanalytical Systems electrochemical detector (LC-3). 'The 7- $\mu \mathrm{m}, 0.46$ $\times 25 \mathrm{~cm}$ Lichrosorb RP-18 column (Unimetrics) was eluted with water/methanol/acetic acid (90:10:1) containing $2 \mathrm{~mm}$ heptanesulfonic acid and $1 \mathrm{~mm}$ sodium

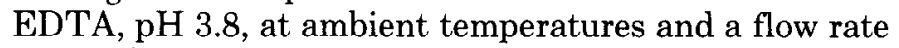
of $1 \mathrm{ml} / \mathrm{min}$; retention time of norepinephrine was about $13 \mathrm{~min}$. The glassy carbon electrode of the detector was maintained at $0.74 \mathrm{~V}$ (versus $\mathrm{Ag} / \mathrm{AgCl}$ ). For norepinephrine the lower limit of detection was 40 to $100 \mathrm{pg}$.

Histology. After taking samples from the cortex, each animal was perfused through the heart with normal saline, followed by buffered formalin. The midbrain was blocked, frozen sectioned, and stained with cresyl violet for Nissl substance. Serial sections were inspected for location of the lesions.

\section{Results}

Electrolytic lesions of the DNAB. The entire procedure was carried out as early as possible in the critical period, which lasts from approximately 3 weeks to 12 weeks of age, with particular sensitivity at 4 to 6 weeks of age (Hubel and Wiesel, 1970). The lesions were made at 3 to 4 weeks of age. Two weeks were allowed following the lesion for presynaptic NA terminals in the visual cortex to degenerate, then the eyelids of one eye of each kitten were sutured closed. Single cell recordings were made at $6 \frac{1 / 2}{2}$ to $7 \frac{1}{2}$ weeks of age after a period of monocular deprivation varying from 7 to 20 days. Details for individual animals are given in Figure 1.

The maximum extent of the lesions and the anteroposterior position of the maximum extent are shown in Figure 2. In all cases the lesion included substantial portions of the medial forebrain bundle, which has the DNAB running in it, and also the lateral hypothalamus. Two lesions bordered on the optic tract, but inspection 


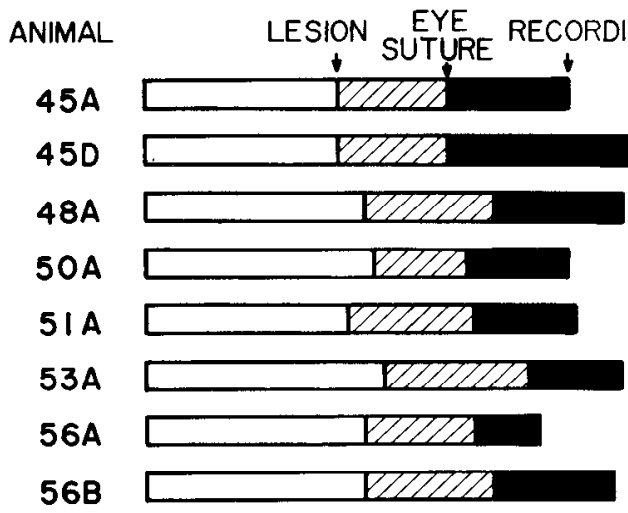

$58 \mathrm{~A}$

66A

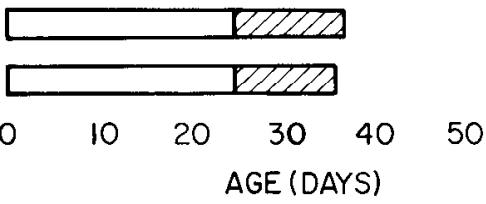

Figure 1. Time course of the procedure and the noradrenaline concentration for animals with electrolytic lesions. Noradrenaline concentration expresses the level on the lesioned side as an average percentage of the level on the intact side. See Table I for individual measurements.

of the lateral geniculate nucleus and the physiological records from the animals suggested that the optic tract was not substantially affected. Other areas included in a few of the lesions were more ventral and medial portions of the hypothalamus and parts of the subthalamus and thalamus.

The effect of the lesions on NA concentration in the cortex is summarized in Table I, with the percentage of depletion for individual animals given in Figure 1. The values for the right cortex $(66 \pm 20 \mathrm{ng} / \mathrm{gm}, n=20)$ are comparable to those observed in the cortex of normal animals of the same age $(60 \pm 27, n=21)$, which are expectedly less than the levels found in adult cats (Bertler and Rosengren, 1959; McGeer et al., 1963; Jonsson and Kasamatsu, 1983) but comparable to the values recently reported for 7-week-old kittens (Jonsson and Kasamatsu, 1983). Somewhat higher control values for kittens of the same age may be due to diurnal variation (Adrien et al., 1982; Bear and Daniels, 1983; Bear et al., 1983), differences in areas of cortex sampled, and subsequent procedures. All three gyri in the left cortex showed, on average, $80 \%$ depletion of NA, when compared to the same gyrus in the same animal in the right (control) cortex (Table I). One animal (53A) exhibited almost $90 \%$ depletion; one animal (45D) showed about $70 \%$ depletion; and most of the other animals, with the exception of $50 \mathrm{~A}$, had depletions somewhere in between these values (Fig. 1). Two control animals (58A and 66A) were assayed for NA depletion 12 days after the lesion, at the time when the earlier eye sutures were performed. Depletion in these animals was comparable to that in the experimental animals (Fig. 1).

The ocular dominance histograms for all eight animals were similar to those generally found in animals deprived monocularly for 7 to 20 days (Fig. 3). Very few cells were dominated by the sutured eye, and few were driven
45

A8.5
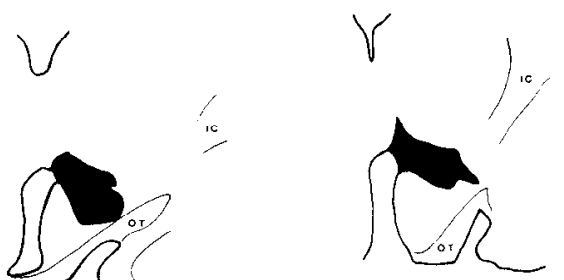

$45 A$

A8
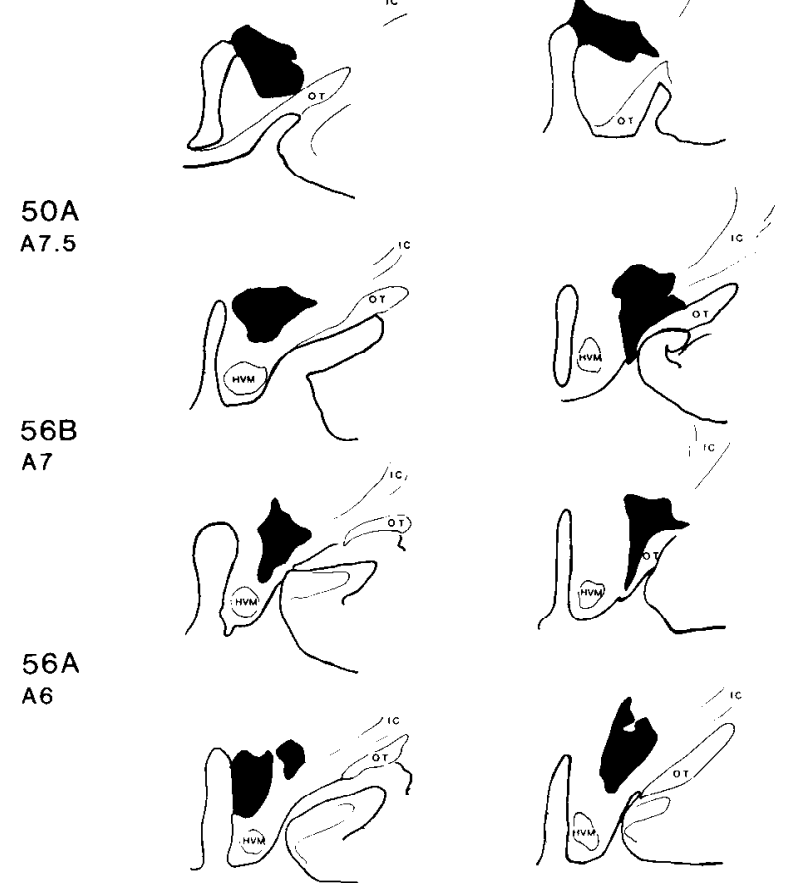

$53 A$

A7

$51 \mathrm{~A}$

A7

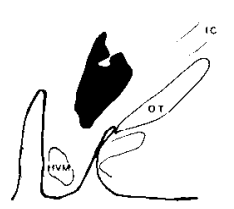

$48 \mathrm{~A}$

A5.5

Figure 2. Maximum extent of 'esions. Lesions are black. Numbers and letters to the side give animal number (e.g., 45A) followed by anteroposterior position of the maximum extent of the lesion (e.g., A8.5). OT, optic tract; $I C$, internal capsule; $H V M$, ventromedial nucleus of the hypothalamus.

TABLE I

Noradrenaline concentrations in animals with electrolytic lesions

Values are given in nanograms per gram. L, lateral gyrus; PL, postlateral gyrus; SS, suprasylvian gyrus; $\mathrm{N}$, sample not analyzed. Samples for $48 \mathrm{~A}$, control cortex, were combined.

\begin{tabular}{|c|c|c|c|c|c|c|c|c|c|}
\hline \multirow{2}{*}{ Animal } & \multicolumn{3}{|c|}{ Control Cortex } & \multicolumn{6}{|c|}{ Experimental Cortex } \\
\hline & $\mathrm{L}$ & PL & SS & & $\mathrm{L}$ & & PL & & SS \\
\hline \multicolumn{10}{|c|}{ Electrolytic lesions } \\
\hline $45 \mathrm{~A}$ & 78 & 86 & 92 & 11 & $(14 \%)$ & & $5(11 \%)$ & 18 & $(20 \%)$ \\
\hline $45 \mathrm{D}$ & 55 & 67 & 93 & $\mathrm{~N}$ & & 33 & $(50 \%)$ & 12 & $(13 \%)$ \\
\hline $48 \mathrm{~A}$ & & 37 & & 3 & $(8 \%)$ & & $(12 \%)$ & 11 & $(30 \%)$ \\
\hline $50 \mathrm{~A}$ & 48 & $\mathbf{N}$ & 71 & 31 & $(65 \%)$ & $\mathrm{N}$ & & $\mathrm{N}$ & \\
\hline $51 \mathrm{~A}$ & 65 & 70 & 101 & 16 & $(24 \%)$ & 21 & $(30 \%)$ & 26 & $(26 \%)$ \\
\hline $53 \mathrm{~A}$ & 69 & 57 & 40 & & $(10 \%)$ & & $5(10 \%)$ & 4.8 & $3(12 \%)$ \\
\hline $56 \mathrm{~A}$ & 41 & 38 & 77 & & $(18 \%)$ & & $2(22 \%)$ & 12 & $(15 \%)$ \\
\hline $56 \mathrm{~B}$ & 49 & 63 & 108 & 17 & $(35 \%)$ & 8 & $(13 \%)$ & 11 & $(10 \%)$ \\
\hline \multicolumn{10}{|c|}{ Control at start of eye suture } \\
\hline $58 \mathrm{~A}$ & 62 & 65 & 62 & 12 & $(19 \%)$ & 12 & $(18 \%)$ & 32 & $(52 \%)$ \\
\hline $66 \mathrm{~A}$ & 82 & 113 & 83 & 11 & $(13 \%)$ & 26 & $(23 \%)$ & $\mathrm{N}$ & \\
\hline
\end{tabular}

binocularly. In animals where the ipsilateral eye was sutured, $82 \%$ (95 of 116) of the cells were driven solely by the contralateral eye. In animals where the contralateral eye was sutured, $71 \%$ ( 75 of 106) of the cells were driven solely by the ipsilateral eye.

6-OHDA lesions of the DNAB. Five animals received a stereotaxically placed injection of 6-OHDA into the DNAB (Fig. 4), with the time course of the experiment 

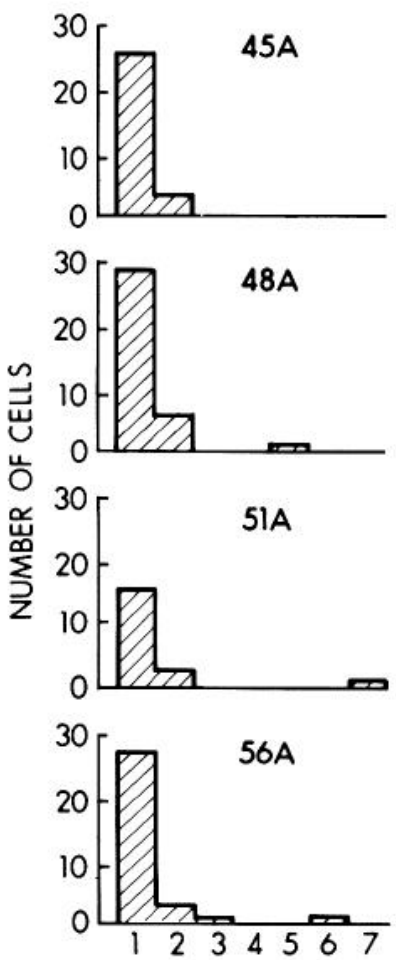

OCULAR DOMINANCE GROUPS

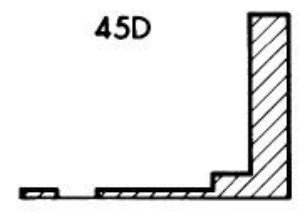

$50 \mathrm{~A}$

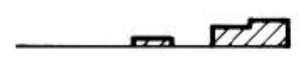

$53 \mathrm{~A}$

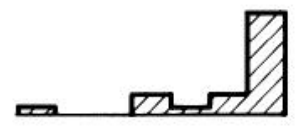

$56 B$

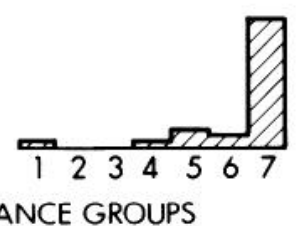

Figure 3. Ocular dominance histograms from animals with electrolytic lesions of the DNAB. Results on the left are from animals with the left (ipsilateral) eye sutured; results on the right are from animals with the right (contralateral) eye sutured.

very much the same as in the electrolytic lesions (Fig. 5 ). In one animal (58C) we suspected that the needle was blocked, and subsequent analysis of NA concentration in the cortex showed no depletion, so this animal was used as a control. The depletion of NA in the left (experimental) cortex was again 70 to $90 \%$ (Table II).

The ocular dominance histograms in these animals were very monocular (Fig. 6). In animals where the ipsilateral eye was sutured, $79 \%$ (57 of 73 ) of the cells were driven solely by the open eye; in animals where the contralateral eye was sutured, $77 \%$ (55 of 71 ) of the cells were driven solely by the open eye. These results are very comparable to data collected from animals with electrolytic lesions of the DNAB.

Comparison with controls. The ocular dominance histograms from both groups of animals were so definitely monocular that measurements from control animals seemed unnecessary. However, recordings were made from the right (control) cortex in two animals with electrolytic lesions and from one animal in which an attempted 6-OHDA lesion did not deplete NA, due to a blocked needle. The results are summarized in Figure 7, which shows that there is no difference between the experimental animals and the control animals and no difference between the animals with electrolytic lesions and the animals with 6-OHDA lesions.

\section{Discussion}

Our results are a clear demonstration that the concentration of NA in the visual cortex can be depleted by 70

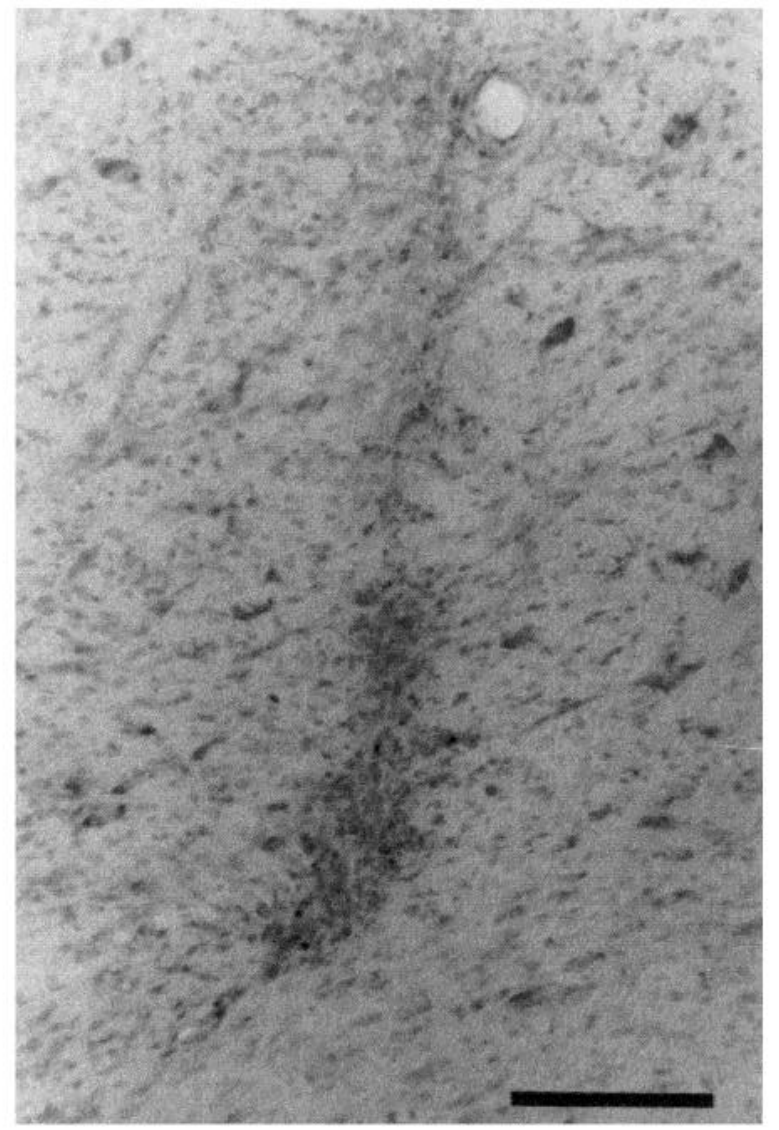

Figure 4. Photomicrograph showing position of the tip of the needle for injection of 6-OHDA in animal 61A. Scale bar $=100$ $\mu \mathrm{m}$.

to $90 \%$ without influencing the effects of monocular deprivation. The interval from lesion to eye suture varied somewhat, between 10 and 16 days; the interval between eye suture and recording varied somewhat, between 7 and 20 days; and the age at which the experiment started varied somewhat, between 21 and 30 days, with essentially the same result. The ocular dominance histogram was definitely monocular, with an average of $77 \%$ (284 of 368) of the cells driven solely by the open eye.

The site chosen for the lesion was the lateral hypothalamus. Although unilateral lesions of the lateral hypothalamus are not nearly as devastating as bilateral lesions, there are some effects. These include neglect of sensory stimuli on the opposite side and circling (Marshall and Teitelbaum, 1974; Wolgin and Teitelbaum, 1978). We did not notice any of these symptoms in our animals. Any form of neglect or inattention, if it had been there, would be expected to prevent the progress of plasticity rather than the reverse. Indeed this was one of the original criticisms of Kasamatsu and Pettigrew's results (1976) - that the behavioral effects of depleting NA might have prevented plasticity through some form of neglect or inattention. Since our results are in the opposite direction, it seems unlikely that some unnoticed behavioral effect of our lesions would have produced them.

When one puts together all of the results from various laboratories at the present time, it is all a little confusing. There are two basic conflicting results. In the first, 

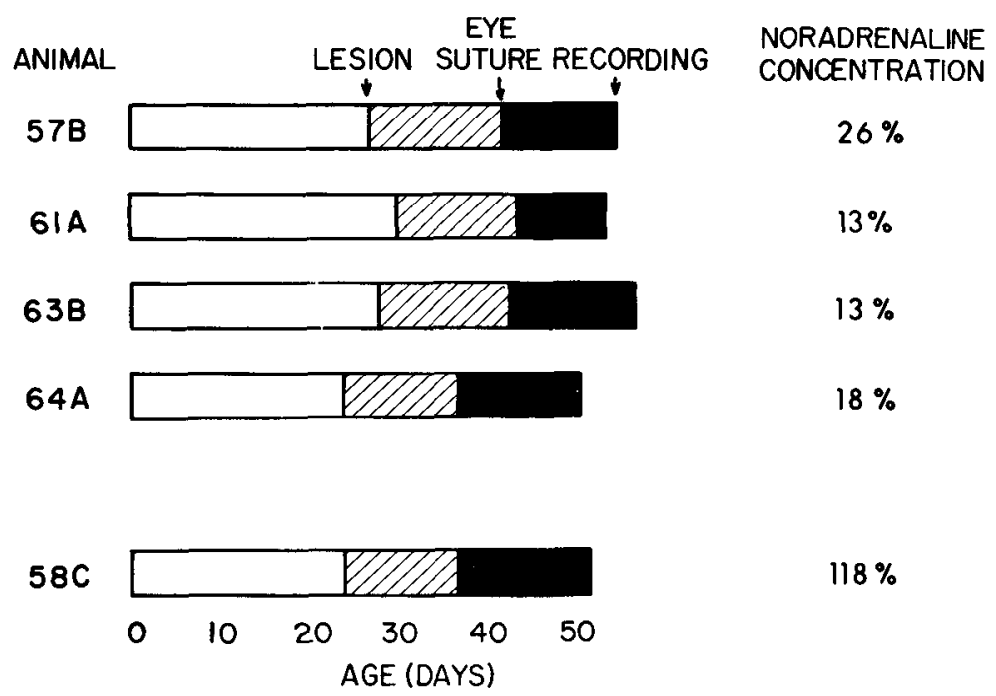

$118 \%$

Figure 5. Time course of procedure and noradrenaline concentration for animals with 6-OHDA lesions. See Table II for individual NA measurements.

TABLE II

Noradrenaline concentrations in animals with 6-OHDA lesions ${ }^{a}$

\begin{tabular}{|c|c|c|c|c|c|c|c|c|}
\hline \multirow{2}{*}{ Animal } & \multicolumn{3}{|c|}{ Control Cortex } & \multicolumn{5}{|c|}{ Experimental Cortex } \\
\hline & $\mathrm{L}$ & $\mathrm{PL}$ & SS & & $\mathrm{L}$ & PL & & SS \\
\hline \multicolumn{9}{|c|}{ 6-OHDA lesions } \\
\hline $57 \mathrm{~B}$ & 68 & 44 & 71 & 15 & $(22 \%)$ & $13 \quad(29 \%)$ & 19 & $(27 \%)$ \\
\hline $61 \mathrm{~A}$ & 99 & 53 & 53 & & $(5 \%)$ & $6.8(13 \%)$ & 11 & $(21 \%)$ \\
\hline $63 \mathrm{~B}$ & 109 & 50 & 68 & $<6$ & $(6 \%)$ & $10 \quad(20 \%)$ & & $6(13 \%)$ \\
\hline $64 \mathrm{~A}$ & 55 & 43 & 60 & 7.9 & $(14 \%)$ & $5.6(13 \%)$ & 17 & $(28 \%)$ \\
\hline \multicolumn{9}{|c|}{ Blocked needle } \\
\hline $58 \mathrm{C}$ & 34 & 53 & 90 & 65 & & 51 & 61 & \\
\hline
\end{tabular}

${ }^{a}$ Details are as in Table I.

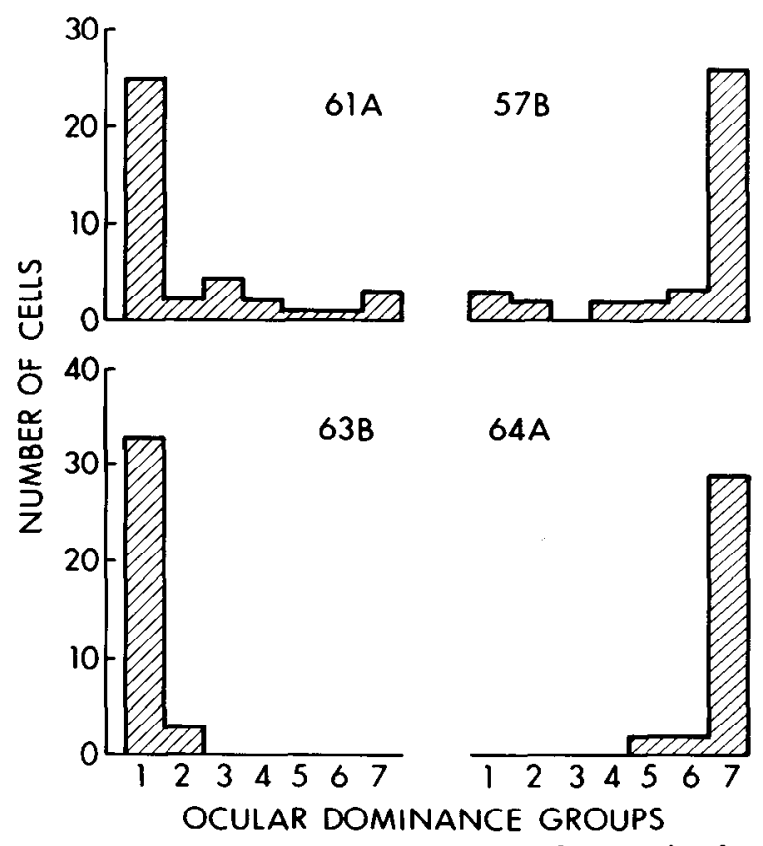

Figure 6. Ocular dominance histograms from animals with 6-OHDA lesions of the DNAB. Details are as in Figure 3.
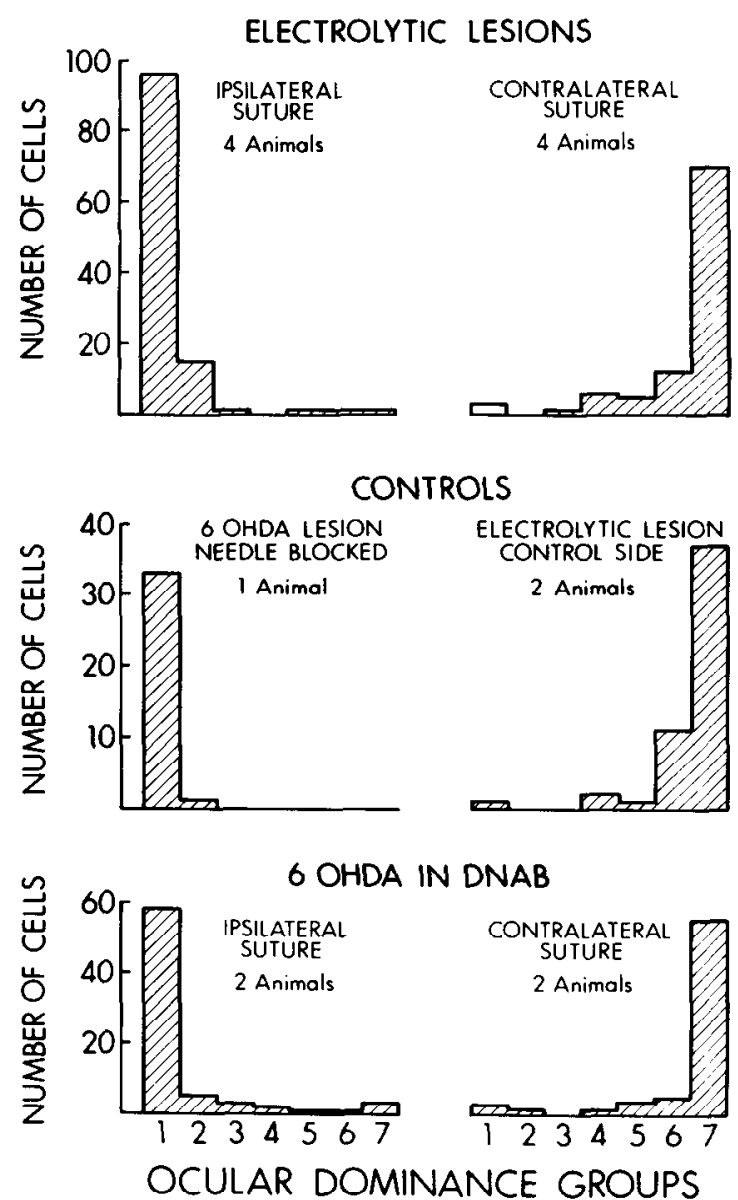

Figure 7. Comparison of the summed ocular dominance histograms from animals with electrolytic lesons, control animals, and animals with 6-OHDA lesions. Details are as in Figures 3 and 6. 
depletion of NA followed by eye suture leads to a monocular ocular dominance histogram. That is, plasticity exists with NA depleted. In the second, depletion of NA followed by eye suture leads to a flatter ocular dominance histogram, with a substantial percentage of binocular cells and a substantial percentage of cells driven by the previously sutured eye. In this case, plasticity is prevented with NA depleted.

The first result is obtained with neonatal intraperitoneal administration of 6-OHDA (Bear and Daniels, 1983), with lesions of the DNAB (this paper), and with lesions of the locus ceruleus (Adrien et al., 1982). The second result is obtained with intracortical administration of 6-OHDA (Kasamatsu et al., 1979; Bear et al., 1983; Daw et al., 1983). Intraventricular administration of 6-OHDA has yielded the first result in one laboratory (Kasamatsu and Pettigrew, 1976, 1979) and the second result in another (Adrien et al., 1982).

Are there any procedural differences that could account for the variety in the results? In most cases the experiment was performed between 3 and 7 weeks of age, and the eyelids were sutured for 1 to 2 weeks, so the length of the monocular deprivation and the timing within the critical period cannot account for it. The extent of NA depletion was also comparable in the various experiments: 70 to $90 \%$ in ours, $80 \%$ in the intracortical 6-OHDA experiments of Bear et al. (1983), 90\% in the neonatal 6-OHDA experiments of Bear and Daniels (1983), 75 to $90 \%$ or more in both the intracortical 6-OHDA and locus ceruleus lesion experiments of Adrien et al. (1982), 80 to $90 \%$ in the intracortical 6-OHDA injections of Kasamatsu et al. (1981), and up to $50 \%$ in the original intraventricular injections of Kasamatsu and Pettigrew (1979). One has to look at these percentages with caution, because physiological recordings and measurement of NA depletion have not always been carried out in the same animal, and the amount of NA in a small sample from a depleted piece of cortex can be very small. However, there is no evidence at the moment that the extent of NA depletion is greater when plasticity is prevented.

Bear and Daniels (1983) suggested that the time course of the experiment might account for the difference between their results and those of Kasamatsu and Pettigrew (1979). Bear and Danicls administered 6-OHDA neonatally, with a 4-week delay before eyelid suture, whereas Kasamatsu and Pettigrew generally sutured the eyelids within a few days of starting 6-OHDA. In our experiments there was a delay of about 2 weeks between lesions of the DNAB and eyelid suture. Bear and Daniels suggested that such a delay might allow for an increase in postsynatic receptors sufficient to compensate for the decrease in presynaptic transmitter content. Such a compensatory increase in postsynaptic receptors has been shown to occur in the rat (Sporn et al., 1976; Jonsson and Hallman, 1978), but there is some doubt whether its magnitude and time course would be large enough to compensate for a reduction of NA by a factor of 10 . This hypothesis is also complicated by the fact that there is a rapid rise of $\beta$-receptors in the normal kitten between birth and 9 weeks of age (Jonsson and Kasamatsu, 1983).

Other possibilities get more involved. 6-OHDA reduces dopamine (DA) as well as NA (Bloom et al., 1969; Uretsky and Iversen, 1970; Breese and Traylor, 1970; for review see Jonsson, 1980) and has some nonspecific effects (Poirier et al., 1972; Butcher et al., 1975). There is not much DA in the visual cortex, and it seems to be depleted by the same amount (40 to $60 \%$, Bear et al., 1983 ) by two methods (intracortical versus neonatal injections of 6-OHDA) that have opposite effects on plasticity.

The possibility that 6-OHDA is acting through some nonspecific effect is intriguing since the two methods that prevent plasticity are the two methods that give 6OHDA the most direct access to the visual cortex. The possibility has never been tested directly by the traditional method of giving 6-OHDA with an NA uptake blocker such as desipramine (Jonsson, 1980). However, one has to account for the result that NA can antagonize the effects of 6-OHDA in these paradigms (Pettigrew and Kasamatsu, 1978; Kasamatsu et al., 1979).

Even more complicated possibilities can be imagined. For example, lesions of the lateral hypothalamus which interrupt the medial forebrain bundle do a lot more than deplete NA in the cortex (Veening et al., 1982). However, it seems premature to discuss the complicated explanations when the simple ones have not been adequately tested.

One intriguing result from the original Kasamatsu series of animals is that, while many cells in the cortex were binocular and many were driven by the deprived eye, the cells in layers of the LGN receiving input from the deprived eye were smaller (Kasamatsu and Pettigrew, 1979; Hitchcock and Hickey, 1982). This might suggest that the effect of 6-OHDA is a local one on the visual cortex rather than an effect on the whole visual system. In our animals we also noticed a cell size difference between the layers of the LGN. It is interesting that this can occur after such short periods of monocular deprivation.

In summary, there is some doubt about whether 6OHDA is acting through the NA system in those situations where it prevents plasticity in the visual cortex. If the NA system is involved, it does not seem to be a simple depletion of NA, but is likely to involve other components of the NA system.

\section{References}

Adrien, J., P. Buisseret, Y. Fregnac, E. Gary-Bobo, M. Imbert, J. -P. Tassin, and Y. Trotter (1982) Noradrénaline et plasticité du cortex visuel du chaton: Un réexamen. C. R. Acad. Sci. Paris III 295: 745-750.

Anand, B. K., and J. R. Brobeck (1951) Hypothalamic control of food intake. Yale J. Biol. Med. 24: 123-140.

Bear, M. F., and J. D. Daniels (1983) The plastic response to monocular deprivation persists in kitten visual cortex after chronic depletion of norepinephrine. J. Neurosci. 3: 407-416.

Bear, M. F., M. A. Paradiso, M. Schwartz, S. B. Nelson, K. M. Carnes, and J. D. Daniels (1983) Two methods of catecholamine depletion in kitten visual cortex yield different effects on plasticity. Nature 302: 245-247.

Berman, N., and N. W. Daw (1977) Comparison of the critical periods for monocular and directional deprivation in kittens. J. Physiol (Lond.) 265: 249-254.

Bertler, A., and E. Rosengren (1959) Occurrence and distribu- 
tion of catecholamines in brain. Acta Physiol. Scand. 47: $350-361$.

Bloom, F. E., S. Algeri, A. Gropetti, A. Revuelta, and E. Costa (1969) Lesions of central norepinephrine terminals with 6 $\mathrm{OH}$-dopamine: Biochemistry and fine structure. Science 166: 1284-1286.

Breese, G. R., and T. D. Traylor (1970) Effect of 6-hydroxydopamine on brain norepinephrine and dopamine: Evidence for selective degeneration of catecholamine neurons. J. Pharmacol. Exp. Ther. 174: 413-420.

Butcher, L. C., G. K. Hodge, and J. C. Schaeffer (1975) Degenerative processes after intraventricular injection of 6-hydroxydopamine. In Chemical Tools in Catecholamine Research, $\mathrm{G}$. Jonsson, T. Malmfors, and C. Sachs, eds., pp. 83-90, Publisher, City.

Chu, N. S., and F. E. Bloom (1974) The catecholamine-containing neurons in the cat dorsolateral pontine tegmentum: Distribution of the cell bodies and some axonal projections. Brain Res. 66: 1-21.

Daw, N. W., and H. J. Wyatt (1976) Kittens reared in a unidirectional environment: Evidence for a critical period. J. Physiol. (Lond.) 257: 155-170.

Daw, N. W., R. K. Rader, T. W. Robertson, and M. Ariel (1983) Effects of 6-hydroxydopamine on visual deprivation in the kitten striate cortex. J. Neurosci. 3: 904-914.

Hitchcock, P. F., and T. L. Hickey (1982) Cell size changes in the lateral geniculate nuclei of normal and monocularly deprived cats treated with 6-hydroxydopamine and norepinephrine. J. Neurosci. 2: 681-686.

Hubel, D. H., and T. N. Wiesel (1959) Receptive fields of single neurons in the cat's striate cortex. J. Physiol. (Lond.) 148. $574-591$.

Hubel, D. H., and T. N. Wiesel (1970) The period of susceptibility to the physiological effects of unilateral eye closure in kittens. J. Physiol. (Lond.) 206: 419-436.

Jonsson, G. (1980) Chemical neurotoxins as denervation tools in neurobiology. Annu. Rev. Neurosci. 3: 169-187.

Jonsson, G., and H. Hallman (1978) Changes in $\beta$-receptor binding sites in rat brain after neonatal 6-hydroxydopamine treatment. Neurosci. Lett. 9: 27-32.

Jonsson, G., and T. Kasamatsu (1983) Maturation of monoamine neurotransmitters and receptors in cat occipital cortex during postnatal critical period. Exp. Brain. Res. 50: 449458.

Kasamatsu, T., and J. D. Pettigrew (1976) Depletion of brain catecholamines: Failure of ocular dominance shift after monocular occlusion in kittens. Science 194: 206-208.

Kasamatsu, T., and J. D. Pettigrew (1979) Preservation of binocularity after monocular deprivation in the striate cortex of kittens treated with 6-OHDA. J. Comp. Neurol. 185: 139162.

Kasamatsu, T., J. D. Pettigrew, and M. Ary (1979) Restoration of visual cortical plasticity by local microperfusion of norepinephrine. J. Comp. Neurol. 185: 163-182.

Kasamatsu, T., T. Itakura, and G. Jonsson (1981) Intracortical spread of exogenous catecholamines: Effective concentration for modifying cortical plasticity. J. Pharmacol. Exp. Ther. 217: 841-850.

Kissinger, P. T., C. Refshauge, R. Dreiling, and R. N. Adams (1973) An electrochemical detector for liquid chromatography with picogram sensitivity. Anal. Lett. 6: 465-477.

Maeda, T., C. Pin, D. Salvert, M. Ligier, and M. Jouvet (1973) Les neurones contenant des catecholamines du tegmentum pontique et leurs voies de projection chez le chat. Brain Res. 57: 119-152.

Marshall, J. F., and P. Teitelbaum (1974) Further analysis of sensory inattention following lateral hypothalamic damage in rats. J. Comp. Physiol. Psychol. 86: 375-395.

McGeer, P. L., E. G. McGeer, and J. A. Wada (1963) Central aromatic amine levels and behaviour. Arch. Neurol. 9: 81-84.

Mitchell, J., and C. J. Coscia (1978) Application of paired-ion high-pressure liquid column chromatography to the analysis of L-3, 4-dihydroxy-phenylalanine metabolites. J. Chromatogr. 145: 295-301.

Morrison, J. H., M. E. Molliver, R. Grzanna, and J. T. Coyle (1981) The intracortical trajectory of the coeruleo-cortical projection in the rat: A tangentially organized cortical afferent. Neuroscience 6: 139-158.

Movshon, J. A., and R. C. Van Sluyters (1981) Visual neural development. Annu. Rev. Psychol. 32: 477-522.

Pettigrew, J. D., and T. Kasamatsu (1978) Local perfusion of noradrenaline maintains visual cortical plasticity. Nature 271: 761-763.

Poirier, L. J., P. Langelier, A. Roberge, R. Boucher, and A. Kitsikis (1972) Non-specific histopathological changes induced by the intracerebral injection of 6-hydroxydopamine (6-OHDA). J. Neurol. Sci. 16: 410-416.

Sherman, S. M., and P. D. Spear (1982) Organization of the visual pathways in normal and visually deprived cats. Physiol. Rev. 62: 738-855.

Sporn, J. R., T. K. Harden, B. B. Wolfe, and P. B. Molinoff (1976) $\beta$-Adrenergic receptor involvement in 6-hydroxydo pamine induced super sensitivity in rat cerebral cortex. Science 194: 624-626.

Teitelbaum, P., and A. N. Epstein (1962) The lateral hypothalamic syndrome. Psychol. Rev. 69: 74-90.

Uretsky, N. J., and L. L. Iversen (1970) Effects of 6-hydroxydopamine on catecholamine containing neurons in the rat brain. J. Neurochem. 17: 269-278.

Veening, J. G., L. W. Swanson, W. M. Cowan, R. Nieuwenhuys, and L. M. G. Geeraedts (1982) The medial forebrain bundle of the rat. II. An autoradiographic study of the topography of the major descending and ascending components. J. Comp. Neurol. 206: 82-108.

Wiesel, T. N., and D. H. Hubel (1963) Single cell responses in striate cortex of kittens deprived of vision in one eye. J. Neurophysiol. 26: 1503-1517.

Wolgin, D. L., and P. Teitelbaum (1978) Role of activation and sensory stimuli in recovery from lateral hypothalamic damage in the cal. J. Comp. Physiol. Psychol. 92: 474-500. 